\title{
The risk allele load accelerates the age-dependent decline in beta cell function
}

\author{
A. Haupt • H. Staiger • S. A. Schäfer • K. Kirchhoff • \\ M. Guthoff • F. Machicao • B. Gallwitz • N. Stefan • \\ H.-U. Häring • A. Fritsche
}

Received: 1 September 2008 / Accepted: 4 December 2008 /Published online: 27 January 2009

(C) Springer-Verlag 2009

\begin{abstract}
Aims/hypothesis Among the novel type 2 diabetes risk loci identified by genome-wide association studies, TCF7L2, HHEX, SLC30A8 and CDKAL1 appear to affect beta cell function. In the present study we examined the effect of these genes' risk alleles on the age-dependent decline in insulin secretion.

Methods The SNPs rs7903146 (TCF7L2), rs7754840 (CDKAL1), rs7923837 (HHEX) and rs13266634 (SLC30A8) were genotyped in 1,412 non-diabetic patients, who were subsequently grouped according to their number of risk alleles. All participants underwent an OGTT. Insulin secretion was assessed by validated indices and proinsulin conversion by calculating $\mathrm{AUC}_{\text {proinsulin }} / \mathrm{AUC}_{\text {insulin }}$.

Results The number of risk alleles revealed a Gaussian distribution, with most participants carrying four risk alleles. Stratification into groups with low (LAL, up to three alleles), median (MAL, four alleles) and high (HAL, five to eight alleles) allele load resulted in MAL and HAL participants displaying significantly lower insulin secretion and proinsulin conversion than LAL participants $(p \leq 0.0014$ and $p=0.0185$, respectively). In the overall cohort, age was negatively
\end{abstract}

Electronic supplementary material The online version of this article (doi:10.1007/s00125-008-1250-2) contains supplementary material, which is available to authorised users.

A. Haupt and H. Staiger contributed equally to this study.

A. Haupt $\cdot$ H. Staiger $\cdot$ S. A. Schäfer $\cdot$ K. Kirchhoff $\cdot$ M. Guthoff $\cdot$

F. Machicao $\cdot$ B. Gallwitz $\cdot$ N. Stefan $\cdot$ H.-U. Häring $(\bowtie) \cdot$

A. Fritsche

Medical Clinic, Department of Internal Medicine IV (Diabetology,

Endocrinology, Nephrology, Angiology, and Clinical Chemistry),

Eberhard Karls University,

Otfried-Müller-Strasse 10,

72076 Tübingen, Germany

e-mail: hans-ulrich.haering@med.uni-tuebingen.de associated with insulin secretion and proinsulin conversion (both $p<0.0001$ ). MAL and HAL participants showed a significantly more pronounced decline in insulin secretion with increasing age than LAL participants $(p \leq 0.0325$; analysis of covariance), and after stratification for BMI this relationship was maintained in obese, but not non-obese, participants. Proinsulin conversion decreased with increasing age in MAL and HAL, but not LAL, participants ( $p \leq 0.0003$ vs $p=0.2)$.

Conclusions/interpretation The risk allele load significantly accelerates the age-dependent decline in beta cell function, and this might be of particular importance in obese people.

Keywords Age $\cdot$ Beta cells · Insulin secretion ·

Proinsulin conversion

\begin{tabular}{ll}
\multicolumn{2}{l}{ Abbreviations } \\
ANCOVA & analysis of covariance \\
HAL & high allele load \\
LAL & low allele load \\
MAL & median allele load \\
SNP & single-nucleotide polymorphism \\
TÜF & Tübingen Family Study for type 2 diabetes
\end{tabular}

\section{Introduction}

Gene-environment interactions are held responsible for the development of type 2 diabetes mellitus. More explicitly, common variation within several genes is thought to confer increased susceptibility to the adverse environmental challenges of the modern civilised world (e.g. high-calorie diets and reduced physical activity) [1]. Using large-scale singlenucleotide polymorphism (SNP) arrays, recent genome-wide 
association studies identified a series of novel type 2 diabetes risk loci [2-6], and the maximum number risk alleles of these genes was subsequently shown to increase the risk of type 2 diabetes approximately 2.2-fold [7]. Among the novel risk loci, TCF7L2, HHEX, SLC3OA 8 and CDKAL1 have been reported to affect diverse beta cell functions, such as overall insulin release, proinsulin-to-insulin conversion, and glucose and incretin responsiveness of the beta cell [8-16].

We have shown earlier that ageing is associated with deteriorating insulin secretion and proinsulin conversion [17]. In order to better understand the role and importance of the risk loci mentioned above in beta cell dysfunction, we asked in this study whether the load of risk alleles of these genes influences the degree of age-dependent decline in beta cell function. To this end, we genotyped 1,412 non-diabetic German participants at increased risk of type 2 diabetes for the genome-wide association-derived diabetes risk SNPs rs7903146 in TCF7L2, rs7754840in CDKAL1, rs7923837 in HHEX and rs13266634 in SLC30A8, divided them according to the number of risk alleles into groups with a low allele load (LAL, zero to three alleles), a median allele load (MAL, four alleles) and a high allele load (HAL, five to eight alleles), and investigated in these groups the associations between age and insulin secretion/proinsulin conversion.

\section{Methods}

Subjects Non-diabetic participants $(n=1412)$ from southern Germany were recruited from the ongoing cross-sectional Tübingen Family Study for type 2 diabetes (TÜF), which currently includes $\sim 2,000$ individuals at an increased risk of diabetes (family history of type 2 diabetes, diagnosis of impaired fasting glycaemia). Type 2 diabetes was an exclusion criterion for the TÜF. More than $99.5 \%$ of TÜF participants are of European ancestry. More information about the TÜF study is given in [18]. Recruitment of the participants was based on the availability of DNA samples and complete data sets (glucose, insulin, C-peptide, and proinsulin measurements). All participants underwent physical examination, medical history assessment, routine blood tests, and an OGTT. The OGTT revealed that $72.9 \%$ of the non-diabetic participants had normal glucose tolerance, 9.9\% had impaired fasting glycaemia, 9.4\% had impaired glucose tolerance and $7.8 \%$ had impaired fasting glycaemia and impaired glucose tolerance. To ensure that we did not miss effects of the risk allele load on glucose tolerance, we left these subgroups in the analysis. The participants were not taking any medication known to affect glucose tolerance or insulin secretion. Characteristics of the overall study population were as follows: age, $39 \pm 13$ years; BMI, $28.5 \pm 7.8 \mathrm{~kg} / \mathrm{m}^{2}$; fasting glucose, $5.12 \pm 0.58 \mathrm{mmol} / \mathrm{l} ; 120 \mathrm{~min}$ glucose, $6.28 \pm$ $1.68 \mathrm{mmol} / \mathrm{l}$; fasting insulin, $62.6 \pm 51.7 \mathrm{pmol} / \mathrm{l} ; 120 \mathrm{~min}$ insulin, $427 \pm 450 \mathrm{pmol} / 1$ (mean $\pm \mathrm{SD})$. Informed written consent was obtained from all participants, and the local ethics committee (Ethikkommission der Medizinischen Fakultät der Universität Tübingen) approved the protocol.

Genotyping DNA was isolated from whole blood using a commercial DNA isolation kit (NucleoSpin; Macherey \& Nagel, Düren, Germany). The SNPs rs7903146 in TCF7L2, rs7754840 in CDKAL1, rs7923837 in HHEX and rs13266634 in SLC30A8 were genotyped using the TaqMan assay (Applied Biosystems, Foster City, CA, USA), as previously described [9]. The overall genotyping success rate was $99.98 \%$. All SNPs were distributed according to Hardy-Weinberg equilibrium. The genotype distribution and risk allele frequencies of the SNPs are given in Table 1.

OGTT After a $12 \mathrm{~h}$ overnight fast, the participants ingested a solution containing $75 \mathrm{~g}$ glucose. Venous blood samples were drawn at $0,30,60,90$ and $120 \mathrm{~min}$, and plasma glucose, insulin, C-peptide and proinsulin concentrations were determined.

Blood analyses Venous plasma glucose was measured using a bedside glucose analyser (glucose oxidase method; Yellow Springs Instruments, Yellow Springs, OH, USA). Plasma insulin and C-peptide levels were determined with a commercial chemiluminescence system (ADVIA Centaur; Siemens Medical Solutions, Fernwald, Germany). Serum proinsulin concentrations were measured using a microparticle enzyme immunoassay (IBL, Hamburg, Germany). The proinsulin assay showed $0 \%$ cross-reactivity with human insulin and C-peptide and the insulin assay showed $0 \%$ cross-reactivity with human proinsulin.

Calculations The AUC of plasma glucose levels during the OGTT was calculated according to the trapezoid method as: $0.5 \times\left[0.5 \times \mathrm{c}(\text { glucose })_{0}+\mathrm{c}(\text { glucose })_{30}+\mathrm{c}(\text { glucose })_{60}+\right.$ $c$ (glucose $\left.)_{90}+0.5 \times \mathrm{c}(\text { glucose })_{120}\right]$. The AUCs of plasma C-peptide, insulin and proinsulin were calculated in a similar way. Insulin secretion was assessed by calculating the ratio

Table 1 Genotype distribution and risk allele frequencies of the analysed SNPs

\begin{tabular}{lllll}
\hline SNP (gene) & \multicolumn{2}{l}{ Genotype } & \multicolumn{2}{c}{ Risk allele frequency } \\
\hline rs7903146 (TCF7L2) & CC & CT & TT & \\
$n$ & 704 & 591 & 117 & 0.29 \\
rs7754840 (CDKAL1) & GG & GC & CC & \\
$n$ & 631 & 629 & 152 & 0.33 \\
rs7923837 (HHEX) & GG & GA & AA & \\
$n$ & 555 & 680 & 177 & 0.63 \\
rs13266634 (SLC30A8) & CC & CT & TT & \\
$n$ & 736 & 552 & 124 & 0.72 \\
\hline
\end{tabular}


Table 2 Associations of risk allele load with anthropometric and metabolic variables

\begin{tabular}{|c|c|c|c|c|c|}
\hline Group & LAL & MAL & HAL & $p_{1}$ value & $p_{2}$ value \\
\hline Number of risk alleles & $0-3$ & 4 & $5-8$ & & \\
\hline$n$ (female/male) & $509(332 / 177)$ & $429(283 / 146)$ & $474(314 / 160)$ & - & - \\
\hline Age (years) & $39 \pm 1$ & $39 \pm 1$ & $39 \pm 1$ & 0.5 & 0.5 \\
\hline BMI $\left(\mathrm{kg} / \mathrm{m}^{2}\right)$ & $29.0 \pm 0.4$ & $27.9 \pm 0.4$ & $28.4 \pm 0.3$ & 0.06 & 0.08 \\
\hline Fasting glucose $(\mathrm{mmol} / \mathrm{l})$ & $5.11 \pm 0.02$ & $5.11 \pm 0.03$ & $5.14 \pm 0.03$ & 0.6 & 0.4 \\
\hline AUC glucose $(\mathrm{mmol} / \mathrm{l})$ & $14.4 \pm 0.1$ & $14.7 \pm 0.2$ & $14.7 \pm 0.2$ & 0.3 & 0.0109 \\
\hline AUC insulin $(\mathrm{pmol} / \mathrm{l})$ & $1,010 \pm 37$ & $872 \pm 32$ & $834 \pm 31$ & 0.0009 & 0.0017 \\
\hline AUC C-peptide (pmol/1) & $4,855 \pm 86$ & $4,417 \pm 81$ & $4,389 \pm 73$ & $<0.0001$ & 0.0004 \\
\hline AUC proinsulin (pmol/1) & $38.3 \pm 2.0$ & $36.9 \pm 1.9$ & $37.6 \pm 1.8$ & 0.7 & 0.6 \\
\hline First-phase insulin (pmol/1) & $1,375 \pm 40$ & $1,185 \pm 35$ & $1,156 \pm 34$ & $<0.0001$ & $<0.0001$ \\
\hline AUC C-peptide/AUC glucose $\left(\times 10^{-9}\right)$ & $340 \pm 5$ & $306 \pm 5$ & $303 \pm 5$ & $<0.0001$ & $<0.0001$ \\
\hline Insulinogenic index $\left(\times 10^{-9}\right)$ & $163 \pm 7$ & $108 \pm 25$ & $148 \pm 13$ & 0.0002 & 0.0014 \\
\hline Disposition index (AU) & $16,365 \pm 377$ & $16,149 \pm 425$ & $16,051 \pm 427$ & 0.4 & 0.0340 \\
\hline AUC proinsulin/AUC insulin & $0.050 \pm 0.003$ & $0.053 \pm 0.002$ & $0.054 \pm 0.002$ & 0.0027 & 0.0185 \\
\hline
\end{tabular}

All data are mean $\pm \mathrm{SE}$ (unadjusted)

$p_{1}, p$ values for comparison of unadjusted data (ANOVA)

$p_{2}, p$ values for comparison of adjusted data; age was adjusted for sex; BMI was adjusted for sex and age; plasma glucose, insulin, C-peptide and proinsulin concentrations and disposition index were adjusted for sex, age and BMI; other insulin secretion variables were adjusted for sex, age, BMI and insulin sensitivity (multivariate linear regression analysis)

AU, arbitrary units

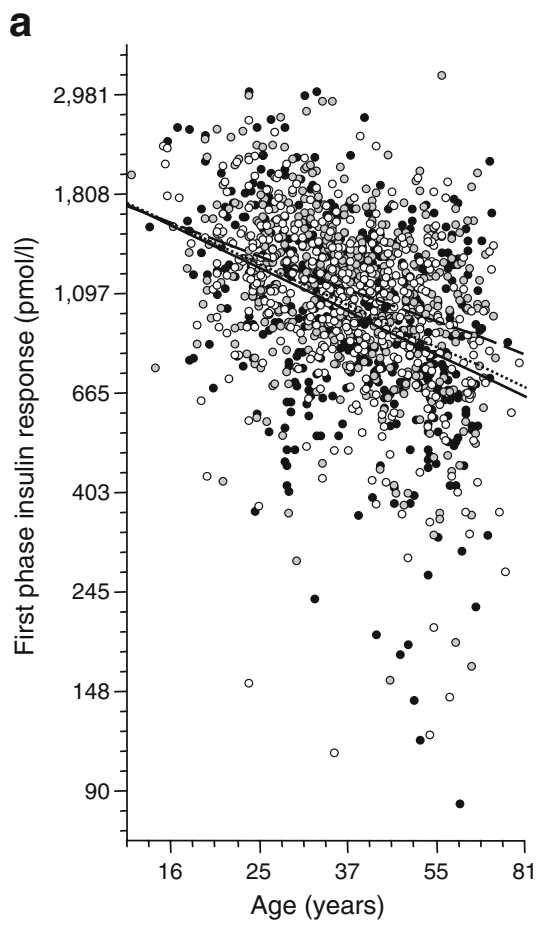

Fig. 1 Effect of risk allele load on the age-dependent decline in insulin secretion. All data were ln-transformed before statistical analysis and plotted using $\ln$ scales. First-phase insulin secretion (a), $\mathrm{AUC}_{\mathrm{C} \text {-peptide }} / \mathrm{AUC}_{\text {glucose }}$ (AUC C-peptide/AUC glucose) (b) and the insulinogenic index (c) were adjusted for sex, BMI and insulin sensitivity by multivariate linear regression modelling (leverage plots shown). Regression lines were compared by ANCOVA with
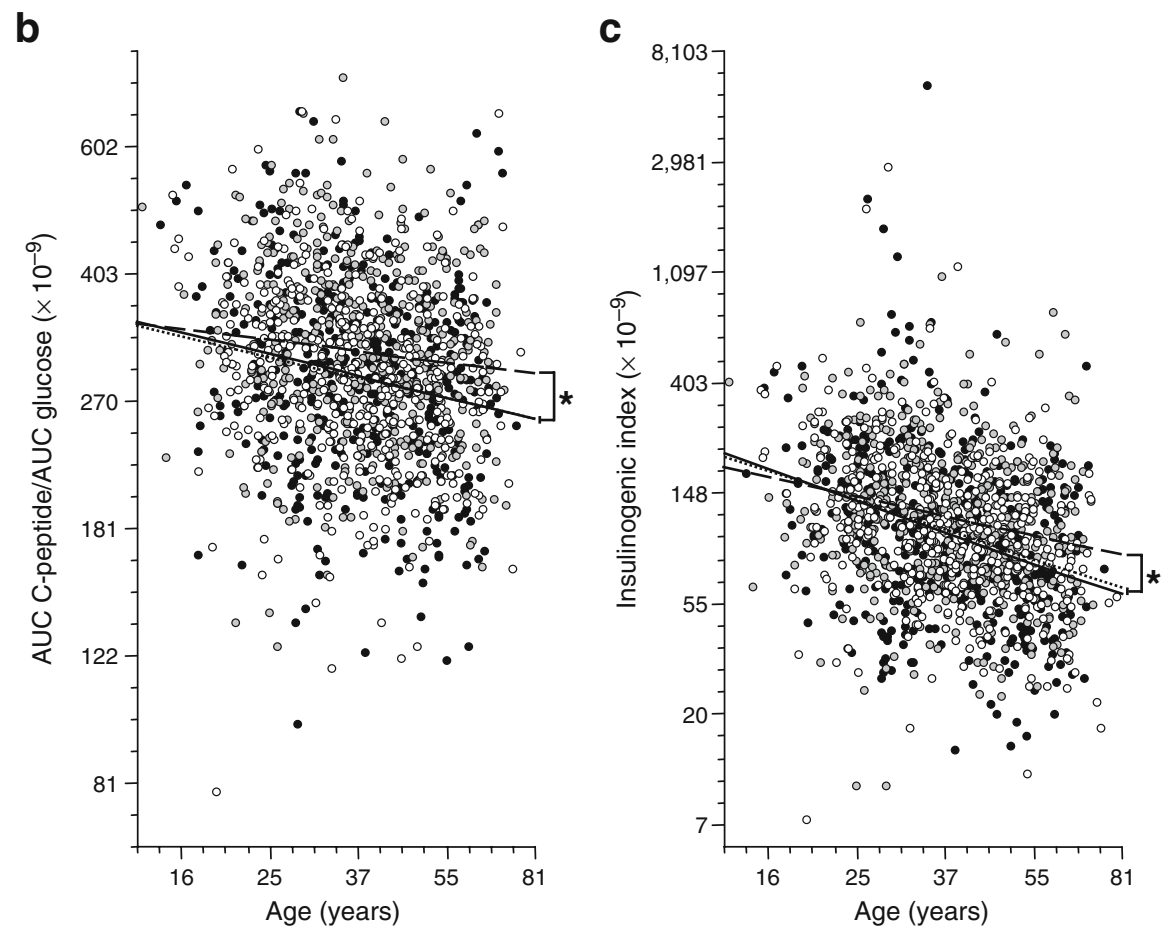

separate slopes $(* p<0.05)$. Grey circles (dashed regression line), LAL $(\mathbf{a}, r=-0.33, p<0.0001 ; \mathbf{b}, r=-0.10, p=0.0243 ; \mathbf{c}, r=-0.22$, $p<0.0001)$; white circles (dotted regression line), MAL (a, $r=-0.40$, $p<0.0001 ; \mathbf{b}, r=-0.19, p<0.0001 ; \mathbf{c}, r=-0.34, p<0.0001)$ black circles (continuous regression line), $\operatorname{HAL}(\mathbf{a}, r=-0.38, p<0.0001$; b, $r=-0.20, p<0.0001$; $\mathbf{c}, r=-0.33, p<0.0001$ ) 
of the AUC of C-peptide divided by the AUC of glucose $\left(\mathrm{AUC}_{\mathrm{C} \text {-peptide }} / \mathrm{AUC}_{\mathrm{glucose}}\right)$. First-phase insulin secretion was estimated from plasma insulin and glucose concentrations as described in [19]: $1,283+1.829 \times \mathrm{c}(\text { insulin })_{30}-$ $138.7 \times \mathrm{c}(\text { glucose })_{30}+3.772 \times \mathrm{c}(\text { insulin })_{0}$, where $\mathrm{c}$ is concentration. The insulinogenic index was calculated as: $\left[\mathrm{c}(\text { insulin })_{30}-\mathrm{c}(\text { insulin })_{0}\right] /\left[\mathrm{c}(\text { glucose })_{30}-\mathrm{c}(\text { glucose })_{0}\right]$. Proinsulin conversion was assessed by calculating the ratio of the AUC of proinsulin divided by the AUC of insulin $\left(\mathrm{AUC}_{\text {proinsulin }} / \mathrm{AUC}_{\text {insulin }}\right)$. Insulin sensitivity was estimated as proposed by Matsuda and DeFronzo [20]: 10,000/ $\left[\mathrm{c}(\text { glucose })_{0} \times \mathrm{c}(\text { insulin })_{0} \times \mathrm{c}(\text { glucose })_{\text {mean }} \times \mathrm{c}(\text { insulin })_{\text {mean }}\right]^{1 / 2}$. The disposition index was calculated as first-phase insulin secretion multiplied by insulin sensitivity.

Statistical analyses Before statistical analysis, all nonnormally distributed variables were $\ln$-transformed in order to approximate normal distribution. Comparisons of unadjusted data between the risk allele groups were performed using one-way ANOVA. Adjustment of indices of beta cell function was performed using multivariate linear regression analysis (least squares method). Leverage plots are shown. Regression lines were compared using analysis of covariance (ANCOVA) with separate slopes. Upon combination of the MAL and HAL groups, the study was sufficiently powered $(1-\beta>0.8)$ to detect differences as small as $2.5 \%$ $\left(\mathrm{AUC}_{\mathrm{C}-\text { peptide }} / \mathrm{AUC}_{\text {glucose }}\right)$ and $6.5 \%\left(\mathrm{AUC}_{\text {proinsulin }} / \mathrm{AUC}_{\text {insulin }}\right)$ between the slopes of the regression lines. Results with $p$ values $<0.05$ were considered statistically significant. The JMP 7.0 statistical software package (SAS Institute, Cary, NC, USA) was used.

\section{Results}

After genotyping of the overall population $(n=1412)$ for the four diabetes risk SNPs in TCF7L2, CDKAL1, HHEX and $S L C 30 A 8$ and grouping of the individuals according to their number of risk alleles, the risk alleles were found to follow a Gaussian distribution, most participants carrying four risk alleles (Electronic supplementary material [ESM] Table 1). The anthropometric and metabolic variables of these groups are presented in ESM Table 1. We then stratified the cohort according to their allele load and defined carriers of up to three alleles as LAL participants, carriers of four alleles as MAL participants, and those with five to eight alleles as HAL participants. For summation of the risk alleles, we weighted each risk allele equally. As shown in Table 2, this

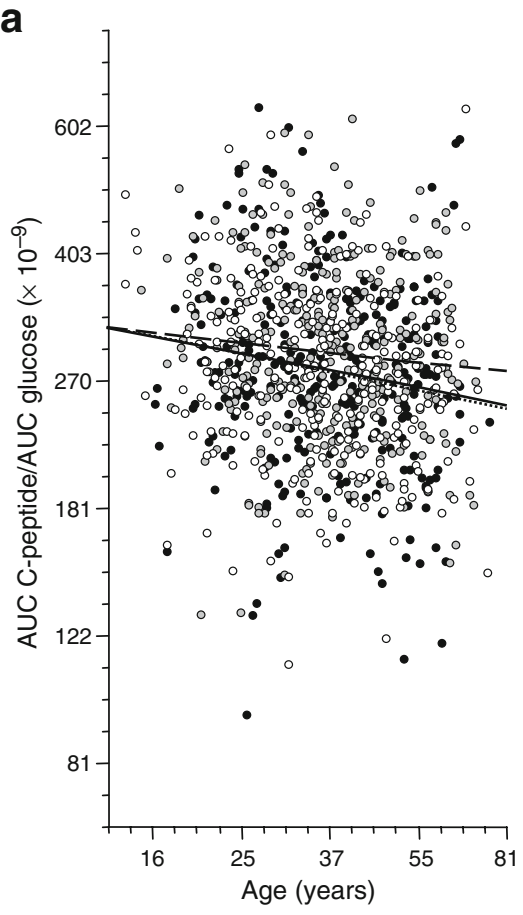

Fig. 2 Effect of risk allele load on the age-dependent decline in insulin secretion in non-obese and obese participants. All data were lntransformed before statistical analysis and plotted using $\ln$ scales. $\mathrm{AUC}_{\mathrm{C} \text {-peptide }} / \mathrm{AUC}_{\text {glucose }}$ (AUC C-peptide/AUC glucose) was adjusted for sex and insulin sensitivity using multivariate linear regression modelling (leverage plots shown). Participants were stratified according to their BMI: non-obese, $\mathrm{BMI}<30 \mathrm{~kg} / \mathrm{m}^{2} \quad(n=966 ;$ a); obese,

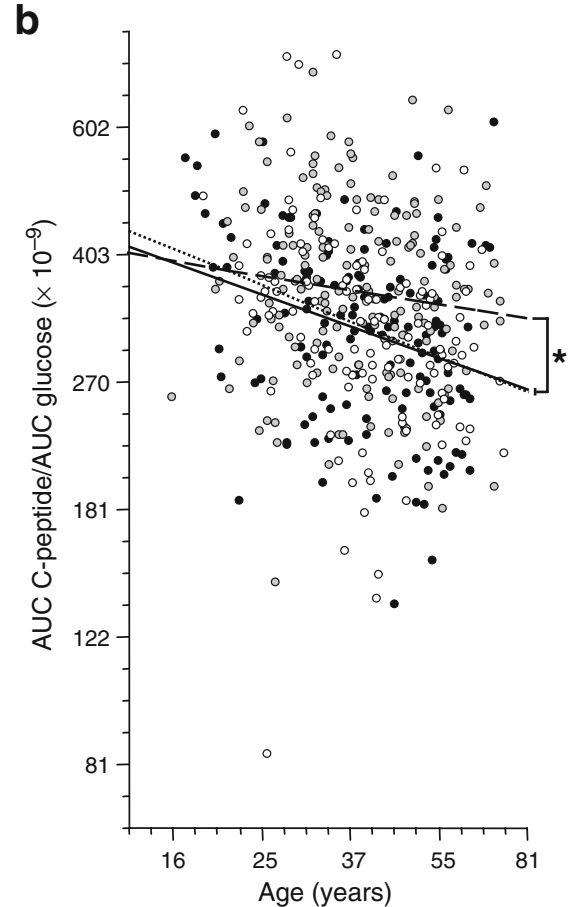

$\mathrm{BMI} \geq 30 \mathrm{~kg} / \mathrm{m}^{2} \quad(n=446 ;$ b $)$. Regression lines were compared by ANCOVA with separate slopes $(* p<0.05)$. Grey circles (dashed regression line), LAL (a, $r=-0.10, p=0.089$ [NS]; b, $r=-0.12, p=0.1[\mathrm{NS}]$ ); white circles (dotted regression line), MAL (a, $r=-0.10, p=0.0018 ; \mathbf{b}, r=-0.27, p=0.0027$ ) black circles (continuous regression line), $\operatorname{HAL}(\mathbf{a}, r=-0.315, p=0.0070 ; \mathbf{b}, r=-0.29, p=0.0004)$ 
procedure resulted in MAL and HAL groups that displayed significantly lower insulin secretion and lower proinsulin conversion $\left(\mathrm{AUC}_{\text {proinsulin }} / \mathrm{AUC}_{\mathrm{insulin}}\right.$ is an inverse measure of proinsulin conversion) than the LAL group $(p \leq 0.0014$ and $p=0.0185$, respectively; adjusted for sex, age, BMI and insulin sensitivity). Furthermore, MAL and HAL participants had a significantly lower disposition index than LAL participants $(p=0.0340$; adjusted for sex, age, and BMI).

In the overall population, age was negatively associated with both insulin secretion and proinsulin conversion (both $p<0.0001$; adjusted for sex, BMI and insulin sensitivity). As shown in Fig. 1a-c, MAL and HAL participants revealed a more pronounced decline in insulin secretion with increasing age than LAL participants, and this effect reached statistical significance using $\mathrm{AUC}_{\mathrm{C} \text {-peptide }} / \mathrm{AUC}_{\text {glucose }}$ and the insulinogenic index as insulin secretion indices (MAL +HAL vs LAL, $p=0.0325$ and $p=0.0262$, respectively; ANCOVA; Fig. 1b, c). Weaker differences in the slopes of the regression lines were seen with the disposition index (ESM Fig. 1). To examine whether the effect of the risk allele load on the age-dependent decline in insulin secretion was due to a skewed distribution between the groups of a single SNP with a predominant effect, we included in addition to the allele load each individual SNP, separately, in the ANCOVA and asked whether this procedure abolished the significant effect of the allele load. By this kind of analysis, we could not detect a predominant effect of any of the individual SNPs (in the dominant or the additive inheritance model) over the effect of the allele load. To assess the influence of obesity status on this allele load effect, we stratified the cohort into non-obese $\left(\mathrm{BMI}<30 \mathrm{~kg} / \mathrm{m}^{2}\right)$ and obese (BMI $\geq 30 \mathrm{~kg} / \mathrm{m}^{2}$ ) groups instead of adjusting for BMI. This showed that the effect of the allele load on $\mathrm{AUC}_{\mathrm{C} \text {-peptide }}$ $\mathrm{AUC}_{\text {glucose }}$ was significant in the obese but not in the nonobese participants ( $p=0.0299$ vs $p=0.1$; ANCOVA; Fig. 2). Furthermore, MAL and HAL participants revealed a decline in proinsulin conversion with increasing age $(p \leq 0.0003$; adjusted for sex, BMI and insulin sensitivity; Fig. 3). However, we did not detect a correlation between age and proinsulin conversion in the LAL group ( $p=0.2$; Fig. 3 ).

\section{Discussion}

In the present study, we examined the effects of the diabetes risk alleles of the TCF7L2, HHEX, SLC3OA8 and CDKAL1 genes on the age-dependent decline in insulin secretion. In accordance with earlier studies that examined each SNP separately [8-16] and a more recent study that reported a cumulative effect of the number of alleles on the risk of type 2 diabetes [7], we found that a high load of risk alleles negatively affected insulin secretion and proinsulin conversion. Furthermore, in the present study we confirmed our

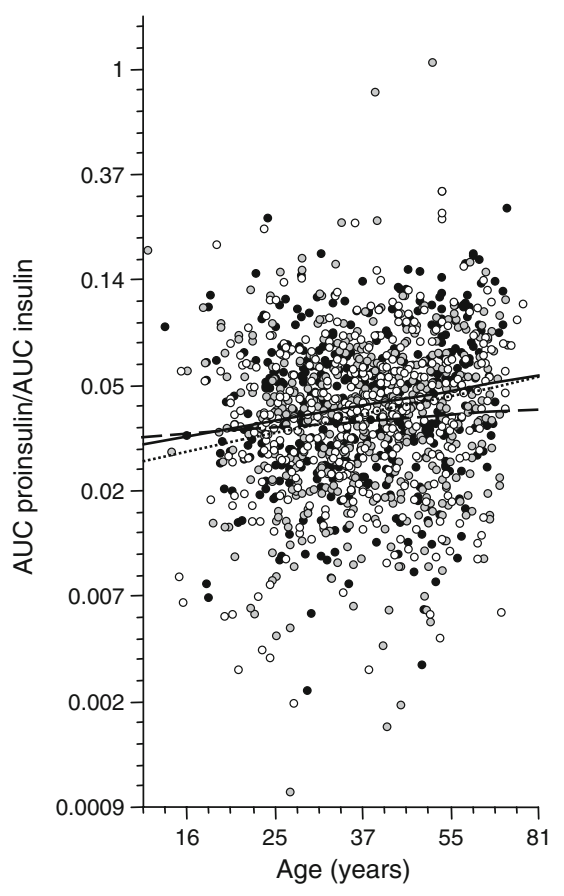

Fig. 3 Effect of risk allele load on the age-dependent decline in proinsulin conversion. All data were $\ln$-transformed before statistical analysis and plotted using $\ln$ scales. $\mathrm{AUC}_{\text {proinsulin }} / \mathrm{AUC}_{\text {insulin }}(\mathrm{AUC}$ proinsulin/AUC insulin) was used as an inverse measure of proinsulin conversion and was adjusted for sex, BMI and insulin sensitivity using multivariate linear regression modelling (leverage plot shown). Grey circles (dashed regression line), LAL $(r=0.06, p=0.2$ [NS]); white circles (dotted regression line), MAL $(r=0.20, p<0.0001)$ black circles (continuous regression line), HAL $(r=0.18, p=0.0003)$

earlier results showing deterioration of insulin secretion and proinsulin conversion with increasing age [17].

Most interestingly however, we demonstrated in this study that a high load of risk alleles significantly accelerates the age-dependent decline in insulin secretion, with 50year-old carriers of five to eight alleles (of the risk loci tested) displaying the same insulin secretion as 70-year-old carriers of only three or fewer alleles. Furthermore, 70-year-old participants with five to eight risk alleles revealed a $13-18 \%$ reduction in insulin secretion compared with carriers of up to three alleles of the same age (depending on the insulin secretion index used). Even though our findings are limited to a non-diabetic cohort, these findings, when extrapolated, suggest that the individual number of risk alleles determines the onset of beta cell failure and type 2 diabetes.

Genetic susceptibility to type 2 diabetes has been reported to be modulated by obesity: SNPs known to affect insulin action were associated with type 2 diabetes in obese participants only, whereas SNPs known to modulate beta cell function (i.e. SNPs in GCK, HNF1A, SLC30A8 and $T C F 7 L 2$ ) were shown to be associated with type 2 diabetes in non-obese participants [21]. We therefore asked whether the effect of the allele load on the age-dependent decline in insulin secretion was influenced by obesity status. Interest- 
ingly, the effect of the allele load was significant only in the obese participants. Thus, our data appear inconsistent with the results of the previous study. However, our study differed from the previous study in several points: (1) we did not assess the effects of the individual gene variants separately, but performed stratification according to the allele load; (2) we included HHEX and CDKAL1, two genes not investigated in the previous study; and (3) we did not investigate the effect of the allele load on the endpoint of diabetes. Even though the studies are not really comparable, both stress the importance of gene-environment interactions in the development of beta cell dysfunction and type 2 diabetes.

Finally, we found that participants with few risk alleles did not display impaired proinsulin conversion with increasing age, whereas participants with a high allele load showed an age-dependent decline in proinsulin conversion (i.e. an increase in the $\mathrm{AUC}_{\text {proinsulin }} / \mathrm{AUC}_{\text {insulin }}$ ratio) with age. Thus, defects in insulin processing, probably caused by reductions in proprotein convertase activities [22], are most probably due to genetics.

Even though we obtained significant results with different, i.e. insulin-and C-peptide-derived, measures of insulin secretion and demonstrated that our study was sufficiently powered to detect even small effect sizes, the moderate sample sizes of our risk allele groups may represent a limitation. Therefore, replication of our findings in larger cohorts is needed.

In conclusion, we show here that the risk allele load significantly accelerates the age-dependent decline in beta cell function and hence might promote beta cell failure and type 2 diabetes. This detrimental effect of the risk alleles might be of particular importance in obese participants.

Acknowledgements We thank all study participants for their cooperation. We gratefully acknowledge the excellent technical assistance of A. Bury, H. Luz, A. Guirguis, M. Weisser and R. Werner. The study was supported by grants from the German Research Foundation (KFO 114/2, Ga 386/9-1).

Duality of interest statement The authors declare that there is no duality of interest associated with this manuscript.

\section{References}

1. Freeman H, Cox RD (2006) Type-2 diabetes: a cocktail of genetic discovery. Hum Mol Genet 15(Spec no. 2):R202-R209

2. Sladek R, Rocheleau G, Rung J et al (2007) A genome-wide association study identifies novel risk loci for type 2 diabetes. Nature 445:881-885

3. Saxena R, Voight BF, Lyssenko V et al (2007) Genome-wide association analysis identifies loci for type 2 diabetes and triglyceride levels. Science 316:1331-1336

4. Zeggini E, Weedon MN, Lindgren CM et al (2007) Replication of genome-wide association signals in UK samples reveals risk loci for type 2 diabetes. Science 316:1336-1341
5. Scott LJ, Mohlke KL, Bonnycastle LL et al (2007) A genomewide association study of type 2 diabetes in Finns detects multiple susceptibility variants. Science 316:1341-1345

6. Zeggini E, Scott LJ, Saxena R et al (2008) Meta-analysis of genome-wide association data and large-scale replication identifies additional susceptibility loci for type 2 diabetes. Nat Genet 40:638-645

7. Lango H, Palmer CN, Morris AD et al (2008) Assessing the combined impact of 18 common genetic variants of modest effect sizes on type 2 diabetes risk. Diabetes 57:3129-3135

8. Staiger H, Machicao F, Stefan N et al (2007) Polymorphisms within novel risk loci for type 2 diabetes determine beta-cell function. PLoS ONE 2:e832

9. Kirchhoff K, Machicao F, Haupt A et al (2008) Polymorphisms in the TCF7L2, CDKAL1 and SLC30A8 genes are associated with impaired proinsulin conversion. Diabetologia 51:597-601

10. Boesgaard TW, Zilinskaite J, Vanttinen M et al (2008) The common SLC30A8 Arg325Trp variant is associated with reduced first-phase insulin release in 846 non-diabetic offspring of type 2 diabetes patients-the EUGENE2 study. Diabetologia 51:816-820

11. Pascoe L, Tura A, Patel SK et al (2007) Common variants of the novel type 2 diabetes genes CDKAL1 and HHEX/IDE are associated with decreased pancreatic beta-cell function. Diabetes $56: 3101-3104$

12. Grarup N, Rose CS, Andersson EA et al (2007) Studies of association of variants near the HHEX, CDKN2A/B, and IGF2BP2 genes with type 2 diabetes and impaired insulin release in 10,705 Danish subjects: validation and extension of genomewide association studies. Diabetes 56:3105-3111

13. Staiger H, Stancakova A, Zilinskaite J et al (2008) A candidate type 2 diabetes polymorphism near the HHEX locus affects acute glucose-stimulated insulin release in European populations: results from the EUGENE2 study. Diabetes 57:514-517

14. Palmer ND, Goodarzi MO, Langefeld CD et al (2008) Quantitative trait analysis of type 2 diabetes susceptibility loci identified from whole genome association studies in the Insulin Resistance Atherosclerosis Family Study. Diabetes 57:1093-1100

15. Stancakova A, Pihlajamaki J, Kuusisto J et al (2008) SNP rs7754840 of CDKAL1 is associated with impaired insulin secretion in non-diabetic offspring of type 2 diabetic subjects (the EUGENE2 study) and in a large sample of men with normal glucose tolerance. J Clin Endocrinol Metab 93:1924-1930

16. Schafer SA, Tschritter O, Machicao F et al (2007) Impaired glucagon-like peptide-1-induced insulin secretion in carriers of transcription factor 7-like 2 (TCF7L2) gene polymorphisms. Diabetologia 50:2443-2450

17. Fritsche A, Madaus A, Stefan N et al (2002) Relationships among age, proinsulin conversion, and beta-cell function in nondiabetic humans. Diabetes 51(Suppl 1):S234-S239

18. Stumvoll M, Tschritter O, Fritsche A et al (2002) Association of the T-G polymorphism in adiponectin (exon 2) with obesity and insulin sensitivity: interaction with family history of type 2 diabetes. Diabetes 51:37-41

19. Stumvoll M, Mitrakou A, Pimenta W et al (2000) Use of the oral glucose tolerance test to assess insulin release and insulin sensitivity. Diabetes Care 23:295-301

20. Matsuda M, DeFronzo RA (1999) Insulin sensitivity indices obtained from oral glucose tolerance testing: comparison with the euglycemic insulin clamp. Diabetes Care 22:1462-1470

21. Cauchi S, Nead KT, Choquet H et al (2008) The genetic susceptibility to type 2 diabetes may be modulated by obesity status: implications for association studies. BMC Med Genet 9:45

22. Steiner DF, Rouille Y, Gong Q, Martin S, Carroll R, Chan SJ (1996) The role of prohormone convertases in insulin biosynthesis: evidence for inherited defects in their action in man and experimental animals. Diabetes Metab 22:94-104 